\title{
Fatores desencadeantes em dermatoses crônicas de crianças com perfis internalizante e externalizante segundo relato materno
}

\author{
Natália Guimarães Dias \\ Faculdade de Filosofia, Ciências e Letras de Penápolis \\ São Paulo, SP, Brasil \\ Márcia Cristina Caserta Gon \\ Caroline Audibert Henrique \\ Lorrana Muriéli Araújo Barros \\ Taís da Costa Calheiros \\ Universidade Estadual de Londrina \\ Paraná, PR, Brasil
}

\begin{abstract}
Resumo
Mães são responsáveis pelos cuidados com a saúde de crianças com dermatoses crônicas. Portanto, faz-se importante conhecer os eventos desencadeantes dos sintomas segundo avaliação materna e se há relação dessa atribuição com o tipo de doença e o perfil comportamental das crianças. Relatos de 13 mães de crianças clínicas (perfil internalizante e/ou externalizante do CBCL) e com dermatite atópica, vitiligo ou psoríase foram analisados. Cinco categorias (fatores ambientais, biológicos e genéticos, situacionais, da interação da criança com mãe/pai e emocionais) foram comparadas com (1) tipo de dermatose e (2) perfil da criança. Houve atribuição de fatores ambientais, biológicos e genéticos (dermatite atópica), situacionais, da interação com mãe/pai e emocionais (vitiligo). Fatores emocionais foram atribuídos às crianças avaliadas como clínicas para os dois perfis e para o internalizante apenas, mas não para o externalizante. Estes resultados podem auxiliar nas decisões médicas quanto ao tratamento e à pertinência da inclusão do acompanhamento psicológico.
\end{abstract}

Palavras-chave: Psicologia médica; Medicina do comportamento; Dermatologia; Cuidadores; Cooperação do paciente.

\section{Triggering factors in chronic dermatoses of children with internalizing and externalizing profiles according to maternal report}

\begin{abstract}
Mothers are responsible for the health care of children with chronic dermatoses. Therefore, it is important to know the triggering events of symptoms according to maternal assessment and if there is a relationship of that attribution to the type of disease and behavioral profile of children. Reports of 13 mothers of clinic children (internalizing and/or externalizing profile of CBCL) and with atopic dermatitis, vitiligo or psoriasis were analyzed. Five categories (environmental, biological and genetic, situational, from the child's interaction with mother/father and emotional factors) were compared by (1) type of dermatosis and (2) child's profile. There was attribution to environmental, biological and genetic (atopic dermatitis), situational, from the interaction with mother/ father and emotional factors (vitiligo). Emotional factors were attributed to children evaluated as clinical by the two profiles and by the internalizing only, but not for the externalizing. These results may help in medical decisions related to the treatment and to the pertinence of including psychological accompaniment.
\end{abstract}

Keywords: Medical psychology; Behavioral medicine; Dermatology; Caregivers; Patient compliance.

\section{Los factores que se desencadenan en las dermatosis crónicas de los niños con perfiles de internalización y externalización según relato materno}

\section{Resumen}

Las madres son responsables por los cuidados con la salud de los niños con dermatosis crónicas. Por lo tanto, es importante conocer los eventos desencadenantes de los síntomas según evaluación materna y si hay relación de esta atribución con el tipo de enfermedad y el perfil de comportamiento de los niños. Relatos de 13 madres de niños clínicos (perfil de internalización y/o de externalización del CBCL) y con dermatitis atópica, vitíligo o psoriasis fueron analizados. Cinco categorías (factores ambientales, biológicos y genéticos, situacionales, de la interacción del niño con madre/padre y emocionales) fueron comparadas con (1) el tipo de dermatosis y (2) el perfil del niño. Hubo atribución de los factores ambientales, biológicos y genéticos (dermatitis atópica), situacionales, de la interacción con madre/padre y emocionales (vitíligo). Los factores emocionales fueron atribuidos a niños evaluados como clínicos para los dos perfiles y de internalización solamente, pero no para el de externalización. Estos resultados pueden ayudar en las decisiones médicas en relación al tratamiento y a la pertinencia de la inclusión del acompañamiento psicológico.

Palabras clave: Psicología médica; Medicina de la conducta; Dermatología; Cuidadores; Cooperación del paciente. 
Dermatite atópica, vitiligo e psoríase são doenças dermatológicas crônicas que têm em comum acometimento patológico da pele, etiologia não totalmente esclarecida e curso crônico e imprevisível com períodos de exacerbação e de remissão. O tratamento corresponde ao uso de medicação tópica e sistêmica e cuidados constantes em relação a produtos químicos, alimentos, vestimentas e outros, aos quais o paciente possa ser fisicamente sensível (Sampaio \& Rivitti, 1998). Embora tenham essas características em comum, a forma de manifestação dos sintomas na pele varia de acordo com a doença. Na dermatite atópica (DA), há coceira de intensidade variada e predominância de lesões em áreas de dobras, onde há intensa sudorese, como pescoço, posterior dos joelhos e anterior dos cotovelos (Amaral, March, \& Sant'Anna, 2012; Silva $\&$ Müller, 2007). No vitiligo (VIT), ocorre a destruição de melanócitos e consequente perda de melanina, o que pode levar à ausência total de pigmentação, chamada de acromia (Silva, Gontijo, Pereira \& Ribeiro, 2007). Já na psoríase (PSO), observam-se lesões avermelhadas recobertas por escamas brancas ou prateadas, com predominância no couro cabeludo, cotovelos e joelhos de adultos, ou em áreas como genitália, região glútea e periumbilical nas crianças (Romiti, Maragno, Arnone, \& Takahashi, 2009).

Essas doenças podem estar presentes desde a infância e afetam a qualidade de vida do paciente ao interferir negativamente nos âmbitos físico, social e emocional de sua vida (Alvarenga \& Caldeira, 2009; Barbarot et al., 2007; Gon, Gon, \& Zazula, 2013; Manzoni et al., 2012; Silva, Fortes, Miot, \& Marques, 2013; Tejada, Mendoza-Sassi, Almeida, Figueiredo, \& Tejada, 2011; Torres, Silva, Magalhães, Morcillo, \& Velho, 2011; Weber, Lorenzini, Reinehr, \& Lovato, 2012). Rotina de tratamento, cuidados constantes com a pele, restrição na participação em atividades esportivas e de lazer e exposição a situações de estigmatização pela visibilidade das lesões podem dificultar a adaptação à doença e tornar-se um evento estressor recorrente (Castro \& Piccinini, 2002; Gon, Rocha, \& Gon, 2005; Pires \& Cestari, 2005). Embora não haja consenso na literatura médica da área sobre a relação entre estresse e surgimento e/ou exacerbação dos sintomas dessas doenças (Souza et al., 2005), estudos têm destacado que eventos estressores podem desencadear os sintomas e/ou prejudicar o seu controle (Barisić-Drusko \& Rucević, 2004; Kurizky \& Mota, 2012; Manolache, 2011; Manolache, PetrescuSeceleanu, \& Benea, 2009; Schwartz, Sepúlveda, \& Quintana, 2009).

Ainda no âmbito emocional, pesquisas indicam que crianças com doença crônica apresentam problemas de comportamento com maior frequência do que outras, sendo destacados, dentre eles, ansiedade, depressão e isolamento (Bennet, 1994; Farrel, Donovan, Turner, \& Walker, 2011; Fontes et al., 2005; Nelms, 1989; Pinquart \& Shen, 2011).

Quando se tratam de crianças com uma doença crônica, os pais assumem a responsabilidade por sua saúde, independentemente da idade dela. Esses participam das consultas, fazendo perguntas ao médico e/ou respondendo às perguntas dele sobre o problema de saúde da criança. Embora a literatura em Psicologia Pediátrica mostre a importância da participação mais ativa da criança nesse processo de comunicação, ainda é a interação verbal médico e cuidador que prevalece durante as consultas e/ou procedimentos como forma principal de troca de informações e de orientações médicas (Tates, Elbers, Meeuwesen, \& Bensing, 2002). Por essa razão, faz-se importante conhecer sobre a perspectiva de cuidadores de crianças com dermatose crônica, quais são os fatores que eles identificam como sendo desencadeantes dos sintomas e verificar se há diferença no conteúdo desses relatos em relação ao tipo de doença e ao perfil comportamental das crianças segundo a sua avaliação.

Esse conhecimento caracteriza-se como importante para que se possa avaliar se estes cuidadores têm clareza quanto à etiologia, ao prognóstico e aos procedimentos médicos que devem ser seguidos para o controle dos sintomas (Alvarenga \& Caldeira, 2009; Ben-Gashir, Seed, \& Hay, 2002). Além disso, a percepção deles quanto ao comportamento da criança como sendo problema ou não e situações de estresse que possam se relacionar ao surgimento e exacerbação dos sintomas poderá auxiliar o médico a identificar a necessidade de acompanhamento de outro profissional da saúde, como psicólogo, nutricionista ou psiquiatra.

Portanto, os objetivos do presente estudo consistiram, a partir de relatos das mães de crianças com doença crônica de pele, em: 1) conhecer quais eventos elas identificam como desencadeantes dos sintomas da doença e 2) verificar se há diferença em seu conteúdo de acordo com o perfil comportamental da criança (i.e., internalizante e externalizante) e o tipo de doença (i.e., dermatite atópica, psoríase e vitiligo).

\section{Método}

\section{Participantes}

Treze mães de crianças de 6 a 12 anos com diagnóstico médico dermatológico de dermatite atópica $(n=6)$, vitiligo $(n=4)$ e psoríase $(n=3)$ as quais foram avaliadas por elas como clínicas para os perfis internalizante, externalizante ou para ambos no 
Child Behavior Checklist (CBCL). Elas se inscreveram para participar do projeto de extensão à comunidade "Atendimento psicológico para crianças com doenças crônicas de pele e seus pais: avaliação e intervenção clínica comportamental" (PROEX 004.001.146.000) na Universidade Estadual de Londrina (UEL). As mães das crianças com dermatite atópica tinham idade entre 32 e 41 anos ( $M=36,6$ anos), eram casadas $(70 \%)$ ou divorciadas $(30 \%)$ e concluíram o Ensino Superior (50\%), Ensino Médio (33,3\%) ou Ensino Fundamental I $(16,7 \%)$. As mães das crianças com vitiligo tinham entre 31 e 46 anos ( $M=35,5$ anos), eram casadas $(60 \%)$ ou divorciadas $(40 \%)$ e concluíram o Ensino Médio (25\%), Ensino Fundamental II (25\%) ou Ensino Fundamental I (50\%). As mães das crianças com psoríase tinham entre 32 e 42 anos de idade $(\mathrm{M}=37,5$ anos), eram casadas $(60 \%)$ ou divorciadas $(40 \%)$ e concluíram o Ensino Superior (25\%), Ensino Médio $(50 \%)$ ou Ensino Fundamental I (25\%).

Entre as crianças com dermatite atópica, quatro eram meninas $(66,7 \%)$ e dois eram meninos $(33,3 \%)$, na faixa etária de 9 a 11 anos e estavam cursando o Ensino Fundamental I (16,7\%) ou o Ensino Fundamental II $(83,3 \%)$. Entre as crianças com vitiligo, três eram meninas $(75 \%)$ e um menino (25\%), na faixa etária de 7 a 12 anos e estavam cursando o Ensino Fundamental I (75\%) ou o Ensino Fundamental II (25\%). Entre as crianças com psoríase, três eram meninas $(75 \%)$ e um menino (25\%), na faixa etária de 6 a 12 anos e estavam cursando o Ensino Fundamental I (75\%) ou o Ensino Fundamental II (25\%).

\section{Instrumentos}

Roteiro de Entrevista Semiestruturada - Utilizado, nessa pesquisa, para levantar os relatos das mães sobre fatores desencadeantes para o surgimento e/ou exacerbação dos sintomas da dermatose crônica da criança. Foi elaborado no projeto de extensão à comunidade, com o objetivo de coletar informações das mães, em entrevista inicial, acerca da interação da criança e de sua família com a doença e o tratamento, assim como possíveis queixas dos familiares sobre o comportamento da criança. A estrutura do roteiro foi composta dos seguintes tópicos para investigação: tratamentos e história da doença; pressões psicológicas sobre os pais; estilo de vida da família; distúrbios de sono; escola; efeitos no comportamento da criança; vida social; relacionamento familiar; apoio fornecido pelos médicos; aspectos financeiros e expectativas para o tratamento.

Child Behavior Checklist for Ages 4-18 (CBCL - Achenbach, 1991) - O CBCL é um instrumento bastante utilizado em estudos de Psicologia da Saúde, nos quais é necessário realizar uma avaliação que pode ser aplicada rapidamente e em diferentes contextos. Tal instrumento é considerado seguro e eficaz na avaliação de comportamentos de crianças por seus cuidadores e tem por objetivo identificar problemas de competência social e de comportamentos de crianças entre quatro e 18 anos, conforme Duarte e Bordin (2000).

Utilizou-se a versão brasileira do instrumento denominada "Inventário de Comportamentos da Infância e Adolescência", traduzida por Bordin, Mari e Caiero (1995), para identificar, segundo a avaliação das mães, a presença ou ausência de problemas de comportamento da criança quanto aos perfis internalizante (INT), externalizante (EXT) ou ambos (INT+EXT). Optou-se pela inclusão dessa última categoria em virtude de que os relatos maternos pudessem ser diferentes quando a criança fosse avaliada como clínica nos dois perfis, simultaneamente, em relação àquela que fosse avaliada em apenas um ou outro.

O instrumento é composto por 138 itens, dos quais 20 avaliam competência social e 118 avaliam problemas de comportamento. No primeiro caso, as questões relacionam-se ao envolvimento da criança/ adolescente em atividades diversas, participação em organizações grupais, relacionamento interpessoal, independência no brincar e desempenho escolar. Ao responderem esses itens, os pais devem comparar os comportamentos de seu filho aos de outras crianças da mesma idade nos últimos seis meses. As questões são divididas em três escalas independentes: escolares, atividades e sociabilidade, sendo que a somatória de pontos delimita a Escala Total de Competência Social e a classificação de tais condutas na categoria clínico (escore $\mathrm{T}<37$ ), limítrofe (escore $37<\mathrm{T}>40$ ) e não clínico (escore $\mathrm{T}>40$ ).

A avaliação sobre problemas de comportamento sumariza descrições de condutas que podem estar presentes ou ausentes na vida da criança/adolescente para que o cuidador pontue a frequência com que ocorrem, de acordo com o critério: 0 (nunca ocorrem), 1 (ocorrem às vezes) e 2 (sempre ocorrem). Essas questões são agrupadas em nove escalas individuais: isolamento social, queixas somáticas, ansiedade/ depressão, problemas sociais, problemas de pensamento, problemas de atenção, comportamento de quebrar regras, comportamento agressivo e problemas sexuais, sendo essa última avaliada apenas para crianças entre quatro e 11 anos de idade. Os dados de algumas dessas escalas são novamente reagrupados, dando origem a dois grupos de perfis: internalizante (escalas de isolamento social, queixas somáticas 
e ansiedade/depressão) e externalizante (escalas do comportamento agressivo e de quebrar regras). Essa somatória delimita o perfil na Escala Total de Problemas de Comportamento como clínico (escore $\mathrm{T}>63$ ), limítrofe (escore $60<\mathrm{T}>63$ ) ou não clínico (escore $\mathrm{T}<60$ ).

\section{Procedimento}

As entrevistas de triagem realizadas por meio do roteiro e a aplicação do CBCL foram conduzidas por três estudantes do quarto e quinto anos do curso de Psicologia da UEL, previamente treinados pela coordenadora do projeto. Elas ocorreram individualmente nas salas da Clínica Psicológica dessa mesma universidade, tiveram duração média de 1 h30min e foram gravadas mediante autorização das participantes. Após receberem informações sobre objetivos do projeto, lerem e assinarem o Termo de Consentimento Livre e Esclarecido, as mães foram entrevistadas pelos estudantes e, em seguida, preencheram o CBCL.

Três estudantes do segundo e terceiro anos do mesmo curso de graduação foram também treinados pela coordenadora do projeto para transcreverem os relatos maternos na íntegra. A primeira autora da pesquisa realizou um recorte de frases dos relatos e o seu agrupamento em categorias. Esse agrupamento foi feito tomando-se por base as partes dos relatos das mães que indicassem fatores desencadeantes para o surgimento e/ou exacerbação dos sintomas da doença de sua criança.

\section{Categorias e Critérios para Inclusão dos Relatos}

Foram organizadas cinco categorias: 1 - Fatores ambientais, 2 - Fatores biológicos e genéticos, 3 - Fatores situacionais relacionados à história de vida da criança, 4 - Fatores da interação da criança com mãe/pai, 5 - Fatores emocionais (duas subcategorias: atribuídos pelas mães e pelos médicos). A Tabela 1 apresenta tais categorias e as respectivas descrições.

\section{Avaliação da Fidedignidade das Categorias Organizadas}

Dois alunos do programa de Mestrado em Análise do Comportamento da Universidade Estadual de Londrina, sem conhecimento prévio acerca do procedimento e dos objetivos do estudo, leram as partes dos relatos selecionadas pela pesquisadora e classificaram-nas de acordo com as categorias previamente organizadas. Fez-se a comparação entre os resultados das duas classificações por meio do cálculo do Índice de Concordância dos Comportamentos (Fagundes, 1983). O ICC adotado foi o de $85 \%$ e obtido para as cinco categorias propostas.

\section{Análise dos Dados}

Foram feitas comparações descritivas considerando-se: (1) a categorização dos relatos, (2) o tipo de dermatose crônica infantil (dermatite atópica, vitiligo e psoríase) e (3) o perfil clínico de acordo com a avaliação das mães no instrumento CBCL (INT, EXT e INT+EXT).

\section{Resultados}

A Tabela 2 apresenta o relato das mães (palavras como substantivos, adjetivos, verbos, etc.) quando questionadas durante a entrevista sobre possíveis fatores desencadeantes para o surgimento e/ou exacerbação dos sintomas da doença. As palavras foram agrupadas em cada uma das cinco categorias, considerando-se a dermatose crônica infantil (DA, PSO e VIT) e os perfis internalizante (INT), externalizante (EXT) e ambos simultaneamente (INT+EXT).

TABELA 1

Descrição das categorias dos relatos das mães

\begin{tabular}{lll}
\hline \multicolumn{1}{c}{ Categorias } & \multicolumn{1}{c}{ Critérios para inclusão dos relatos } \\
\hline 1. Fatores ambientais & $\begin{array}{l}\text { Produtos de limpeza, animais de estimação, peças de vestuário, temperatura, alimentação, } \\
\text { pomada, medicação, medicação alternativa. } \\
\text { Bactérias, vírus, fatores congênitos, hereditariedade ou predisposição à doença. }\end{array}$ \\
$\begin{array}{lll}\text { 2. Fatores biológicos e genéticos } & \text { Eventos marcantes, únicos e pontuais da vida da criança ou da família já ocorridos, como: } \\
\text { 3. Fatores situacionais relacionados à história } \\
\text { de vida da criança }\end{array}$ & $\begin{array}{l}\text { Práticas educativas maternas e/ou paternas para o controle do comportamento da criança. } \\
\text { 4. Fatores da interação da criança com mãe/pai }\end{array}$ \\
$\begin{array}{ll}\text { 5A. Fatores emocionais atribuídos pelas mães } \\
\text { 5B. Fatores emocionais atribuídos por médicos ou } \\
\text { outros profissionais de saúde }\end{array}$ & $\begin{array}{l}\text { Sentimentos, pensamentos, emoções. } \\
\text { Sentimentos, pensamentos, emoções. }\end{array}$ \\
\hline
\end{tabular}


TABELA 2

Palavras extraídas dos relatos das mães e agrupadas nas cinco categorias

\begin{tabular}{|c|c|c|c|c|c|c|c|}
\hline Doença & Perfil & $C 1$ & $C 2$ & $C 3$ & $C 4$ & C5A & $C 5 B$ \\
\hline \multirow[t]{3}{*}{ DA } & $\begin{array}{l}\text { INT } \\
(n=1)\end{array}$ & Alimentos & Bactéria & - & - & - & - \\
\hline & $\begin{array}{l}\text { EXT } \\
(n=2)\end{array}$ & $\begin{array}{l}\text { Alimentação } \\
\text { Pó } \\
\text { Cobertor }\end{array}$ & $\begin{array}{l}\text { Alérgico } \\
\text { Problema respiratório }\end{array}$ & $\begin{array}{l}\text { Morte da avó } \\
\text { Gravidez }\end{array}$ & Pai não está & - & Emocional \\
\hline & $\begin{array}{l}\mathrm{INT}+\mathrm{EXT} \\
(\mathrm{n}=3)\end{array}$ & $\begin{array}{l}\text { Alimentar } \\
\text { Cortina } \\
\text { Carpete } \\
\text { Tapete } \\
\text { Animais } \\
\text { Poeira } \\
\text { Pomada } \\
\text { Calor } \\
\text { Verão } \\
\text { Transpiração } \\
\text { Banho } \\
\text { Amaciante } \\
\text { Roupas }\end{array}$ & $\begin{array}{l}\text { Família é alérgica } \\
\text { Tia tem DA }\end{array}$ & $\begin{array}{l}\text { Conheceu } \\
\text { o pai }\end{array}$ & $\begin{array}{l}\text { Brigar } \\
\text { Levar um tapa } \\
\text { Pegar no pé } \\
\text { Falar muito }\end{array}$ & $\begin{array}{l}\text { Nervoso } \\
\text { Triste } \\
\text { Ansiosa } \\
\text { Agressiva } \\
\text { Preocupada } \\
\text { Saudade } \\
\text { Emocional } \\
\text { Agitação }\end{array}$ & $\begin{array}{l}\text { Psicológico } \\
\text { Ansiedade }\end{array}$ \\
\hline \multirow[t]{3}{*}{ VIT } & $\begin{array}{l}\text { INT } \\
(n=2)\end{array}$ & Inverno & Sangue & $\begin{array}{l}\text { Piscina } \\
\text { Acidente } \\
\text { Avô morreu }\end{array}$ & $\begin{array}{l}\text { Pai bebe } \\
\text { Brigar }\end{array}$ & Emocional & Emocional \\
\hline & $\begin{array}{l}\text { EXT } \\
(n=1)\end{array}$ & - & - & Gravidez & Briga & $\begin{array}{l}\text { Agitada } \\
\text { Nervosa } \\
\text { Frustrada } \\
\text { Psicológico } \\
\text { Temperamento }\end{array}$ & $\begin{array}{l}\text { Estresse } \\
\text { Psicológico }\end{array}$ \\
\hline & $\begin{array}{l}\mathrm{INT}+\mathrm{EXT} \\
(\mathrm{n}=1)\end{array}$ & - & - & Gravidez & Falar & $\begin{array}{l}\text { Nervosa } \\
\text { Irritada }\end{array}$ & $\begin{array}{l}\text { Nervosa } \\
\text { Irritada }\end{array}$ \\
\hline \multirow[t]{2}{*}{ PSO* } & $\operatorname{EXT}(n=1)$ & $\begin{array}{l}\text { Inverno } \\
\text { Alimentos } \\
\text { Bichos de pelúcia }\end{array}$ & $\begin{array}{l}\text { Genético } \\
\text { Pai e tia têm psoríase }\end{array}$ & - & - & Ansiosa & $\begin{array}{l}\text { Psicológica } \\
\text { Nervosa }\end{array}$ \\
\hline & $\begin{array}{l}\mathrm{INT}+\mathrm{EXT} \\
(\mathrm{n}=2)\end{array}$ & $\begin{array}{l}\text { Tênis } \\
\text { Meia }\end{array}$ & - & $\begin{array}{l}\text { Separar e } \\
\text { voltar (pais) } \\
\text { Agressão } \\
\text { Visita ao colégio }\end{array}$ & $\begin{array}{l}\text { Pai bebe } \\
\text { Ficar sem ver } \\
\text { o pai }\end{array}$ & $\begin{array}{l}\text { Tensão } \\
\text { Nervoso } \\
\text { Estresse }\end{array}$ & $\begin{array}{l}\text { Nervoso } \\
\text { Estresse }\end{array}$ \\
\hline
\end{tabular}

* Não há inclusão de crianças com psoríase associadas a perfil internalizante, pois as mães desses participantes não os avaliaram como clínicos isoladamente para esse perfil.

Observa-se que as mães de crianças com DA relataram como fatores desencadeantes para o surgimento e/ou exacerbação dos sintomas os ambientais (C1) e os biológicos e genéticos (C2); as mães de crianças com VIT atribuíram como fatores desencadeantes os situacionais (C3), a interação da criança com mãe/ pai (C4) e os emocionais, citados por elas (C5A) e pelos médicos (C5B); as mães de crianças com PSO mencionaram como fatores desencadeantes os ambientais $(\mathrm{C} 1)$ e os emocionais, atribuídos por elas (C5A) e pelos médicos (C5B).

Todas as mães de crianças com DA, VIT e PSO que avaliaram os filhos como clínicos para perfil internalizante e externalizante, simultaneamente, relacionaram o surgimento e/ou exacerbação dos sintomas da dermatose da criança a fatores situacionais (C3), fatores da interação da criança com mãe/pai (C4) e fatores emocionais (mães - 5A e médicos - 5B).

\section{Discussão}

$\mathrm{Na}$ Categoria 1 (fatores ambientais), os relatos das mães de crianças com DA a respeito de fatores desencadeantes do surgimento e/ou exacerbação dos sintomas estão de acordo com o que está descrito na literatura de doenças crônicas de pele, independentemente do perfil clínico da criança encontrado no CBCL. A literatura destaca, como possíveis fatores ambientais desencadeantes da DA, o contato com aeroalérgenos, colonizações bacterianas, 
produtos de limpeza e higiene, mudanças climáticas, alimentação, deficiência de vitamina $\mathrm{D}$ e presença de distúrbios emocionais nos cuidadores de crianças com dermatoses (Gon et al., 2013; Guibas, Gregoriou, Makris, Rigopoulos, \& Chliva, 2013; Lipnharski, Bessa, Azevedo, Bonamigo, \& Quinto, 2013; Manzoni et al., 2013; Mesquita, Costa, \& Igreja, 2013; Rodrigues, Montealegre, Pires, Melo, \& Hahnstadt, 2011). Esses fatores foram identificados nos relatos das mães de crianças com DA, tais como: "verão assim é muito frequente devido à transpiração na dobra", "alimentos como chocolate, peixes e frutos do mar" (sic).

Destacam-se também os cuidados necessários com a pele para prevenir ou minimizar a intensidade dos sintomas, como, por exemplo, banhos rápidos, com água morna e de imersão, aplicação de medicamentos tópicos com a pele levemente úmida, redução de contato com substâncias irritantes, uso de roupas claras, leves e de algodão, manutenção da temperatura do ambiente estável, diminuição da prática de atividades físicas que produzam sudorese, bem como redução do uso de carpetes, cortinas e objetos de pelúcia (Pires \& Cestari, 2005).

Os fatores ambientais desencadeantes para o VIT foram descritos apenas na narração de uma das mães, a qual avaliou seu filho como clínico para perfil internalizante. Essa mãe relacionou o início ou agravamento da lesão da pele da criança ao inverno. Pode-se hipotetizar, a partir do relato dela, que ela tenha atribuído a piora da lesão da pele nesse período em função da necessidade de a criança permanecer por um tempo sob o sol após aplicar a pomada, o que acontece com menor frequência no inverno, resultando em uma maior dificuldade de manutenção do tratamento médico. Essa questão é abordada por Palermo (2010), na ocasião vice-presidente da Sociedade Brasileira de Cirurgia Dermatológica [SBCD], porém justificada de maneira distinta. Segundo ela, esse agravamento estaria relacionado a três fatores: menor ativação das células que produzem o manto hidrolipídico da pele, menor sudorese e ao hábito de tomar banhos quentes e demorados, o que diminui a proteção natural da pele, deixando-a mais seca e suscetível ao contato com alérgenos e outros.

Entre as palavras selecionadas nos relatos de mães de crianças com PSO, foram identificadas semelhanças com fatores correlacionados à piora dos sintomas de acordo com a literatura médica, como o inverno e a ingestão de determinados alimentos (Brufau, Berná, Redondo, Andreo, \& Gras, 2010). Porém, os outros fatores mencionados por elas, como uso de tênis e meia e contato com bichos de pelúcia não são referenciados como desencadeantes. É possível que essas verbalizações tenham ocorrido como consequência do estabelecimento de relações temporais e não de relações de dependência direta entre eventos, como no caso de a criança ter usado calçado fechado (necessário em dias frios) e as lesões do pé terem piorado.

A atribuição de fatores desencadeantes de sintomas a fatores biológicos e genéticos (Categoria 2) foi encontrada nos relatos de mães de crianças com DA, independentemente do perfil comportamental. Esses fatores foram apontados por elas como alergia, problema respiratório e histórico de alergia ou dermatose na família. A referência a alergias e problemas respiratórios era esperada, pois, de acordo com a literatura da área, tais manifestações fazem parte do quadro crônico da doença (Chacha, Ayache, Andrade, Sugai, \& Wiziack, 2009).

Similarmente ao observado na Categoria 1, foi obtido relato de uma das mães de crianças com VIT e perfil internalizante compatível com a Categoria 2. Tal mãe relatou somente fatores da fisiologia do organismo como desencadeantes da doença, o que pode ser observado na seguinte transcrição: "tô esperando retorno com o médico para ver se nesses exames de sangue dá algum problema e daí tem a explicação para saber por que saiu esse vitiligo" (sic). A respeito da atribuição da fisiologia como variável envolvida no desencadeamento de sintomas, estudos têm demonstrado a possibilidade de que mecanismos genéticos, autoimunes, metabólicos e neurais estejam relacionados ao surgimento e/ou agravamento do VIT, mas sua etiologia não está elucidada e não pode ser identificada em exames sanguíneos (Rosa \& Natali, 2009; Sant'Anna, Giovanetti, Castanho, Bazhumi, \& La Selva, 2003).

Entre as mães de crianças com PSO, foram obtidos relatos de atribuição a fatores desencadeantes genéticos (histórico na família) apenas para uma das mães, a qual avaliou o filho como clínico para perfil externalizante. Antecedentes familiares na etiologia da PSO têm sido encontrados em $50 \%$ dos pacientes que manifestam ou manifestaram os sintomas ainda na infância (Brufau et al., 2010), o que pode corroborar, parcialmente, essa atribuição.

Destarte, mães de crianças com doenças crônicas de pele identificaram corretamente (à exceção de alguns relatos já discutidos) fatores ambientais (Categoria 1) e biológicos e genéticos (Categoria 2) relacionados ao surgimento e piora dos sintomas. No entanto, relataram também a atribuição de fatores desencadeantes a outros que não biológicos e/ou ambientais, como fatores situacionais referentes à história de vida 
da criança (Categoria 3), interação da criança com mãe/pai (Categoria 4) e fatores emocionais (Categoria 5, relatados por elas e por médicos ou outros profissionais de saúde), os quais serão discutidos a seguir.

$\mathrm{Na}$ Categoria 3 (fatores situacionais relacionados à história de vida da criança), foram obtidos relatos de mães de crianças com DA cujo comportamento foi avaliado como clínico para os dois perfis e para o perfil externalizante, isoladamente. Nessas verbalizações, foram relatadas situações pontuais, como a morte de um membro da família, gravidez e o momento de conhecer o próprio pai. Esse primeiro acontecimento também foi descrito por Souza et al. (2005) como um dos fatores responsáveis pela dermatose segundo adultos e/ou cuidadores.

Notou-se nos relatos de mães de crianças com VIT, independentemente do perfil da criança pelo CBCL, a referência a fatores situacionais (por exemplo, morte de um membro da família, gravidez e acidente), sendo esses condizentes com a literatura sobre fatores desencadeantes de VIT em crianças (Barisić-Drusko \& Rucević, 2004; Manolache, 2011; Manolache et al., 2009; Schwartz et al., 2009).

Mães de crianças com PSO avaliadas como clínicas para os perfis internalizante e externalizante, simultaneamente, apresentaram relatos compatíveis com a Categoria 3, mas que se diferenciaram das situações descritas para as crianças com DA e VIT. Foram descritos eventos como a separação ou reconciliação dos pais, violência familiar e visita à escola da criança. Entre crianças com PSO, BarisićDrusko e Rucević (2004) relataram a ocorrência de atribuições referentes à morte ou afastamento de familiares, divórcio dos pais e outros fatores.

As mesmas mães que atribuíram como fatores desencadeantes para o surgimento e/ou exacerbação dos sintomas da doença os situacionais também apresentaram relatos de atribuição a fatores da interação da criança com mãe/pai (Categoria 4). Sendo assim, mães de crianças com DA avaliadas com os dois perfis e apenas externalizante citaram brigas, "pegar no pé", "levar um tapa", "falar muito" e o afastamento do pai como possíveis responsáveis pela piora dos sintomas. Esse dado fora também encontrado por Ludwig et al. (2008) e Souza et al. (2005), os quais relatam a influência de interações familiares na progressão da DA.

Mães de crianças com VIT, independentemente do perfil comportamental do filho no CBCL, também emitiram relatos compatíveis com a Categoria 4, tendo citado alcoolismo paterno e brigas como fatores desencadeantes. Barisić-Drusko e Rucević (2004) observaram que, entre crianças com VIT, os fatores citados com mais frequência $(56,9 \%)$ são aqueles relacionados aos psicológicos, definidos pelos autores como agudos (morte de um dos pais ou amigo, nascimento de uma criança na família e mudança de escola ou de casa) ou crônicos (abuso de álcool na família, desarmonia frequente na família ou na escola e divórcio dos pais).

Finalmente, identificou-se a atribuição de fatores desencadeantes àqueles da interação da criança com mãe/pai apenas entre as mães de crianças com PSO avaliadas para os dois perfis, ao mesmo tempo. Entre eles, citou-se: dificuldade de relacionamento familiar, alcoolismo e afastamento paterno, apontados também por Barisić-Drusko e Rucević (2004) em 11,4\% dos relatos nessa população. Questões familiares estressoras (morte, doença, disputas) são comumente descritas como fatores desencadeantes em crianças com PSO (Manolache, 2011).

Portanto, mesmo existindo diferenças entre os sintomas das doenças investigadas no presente estudo e seus fatores desencadeantes, há relatos em comum sobre alterações ambientais negativas, de estresse agudo, como restrição de contato social da criança com pessoas importantes para o seu desenvolvimento (e.g., morte, separação ou afastamento de familiares) e de estresse prolongado (e.g., convívio com alcoolismo, brigas e discussões entre membros da família). Por tratar-se de situações de exposição a eventos estressores por tempo prolongado, as crianças podem desenvolver respostas de ansiedade, depressão, raiva e frustração (Gon et al., 2013) que as classificam, sobretudo, no perfil internalizante.

Sobre os relatos incluídos na Categoria 5 (fatores emocionais atribuídos pelas mães, por médicos ou outros profissionais de saúde), verificou-se que as mães de crianças com VIT e PSO, independentemente do perfil no CBCL, e as de crianças com DA avaliadas como clínicas para os dois perfis, simultaneamente, atribuíram o aparecimento e/ou exacerbação dos sintomas aos fatores emocionais (Categoria 5A). Todas essas mães também relataram tal atribuição como tendo sido feita por médicos ou outros profissionais de saúde (Categoria 5B).

Além disso, as mães que avaliaram os filhos como clínicos para os dois perfis, simultaneamente, e para o perfil internalizante, isoladamente, identificaram o alcoolismo ou separação do pai e briga com mãe/ pai, por exemplo, como prováveis situações que desencadearam os sintomas da doença e, ao mesmo tempo, indicaram os sentimentos, como raiva, tristeza ou ansiedade, enquanto fatores desencadeantes também. Tal relação é sugerida no seguinte relato: "porque ela 
não conhecia o pai, agora esse ano que ela conheceu. Então tem época que ela fica assim triste, que ela sente saudade... então eu já reparei que quando ela está muito nervosa, ansiosa com alguma coisa, ai que dana essa coceira na barriga e onde tem as marcas" (sic). Embora a emoção, denominada de tristeza ou raiva, por exemplo, possa ser considerada como um dos fatores desencadeantes dos sintomas da doença de pele, essa atribuição, quando feita sem a identificação adequada de eventos que contribuíram para que ela se sentisse triste ou raivosa, poderá dificultar a compreensão das mães sobre o problema, as quais, muitas vezes, tendem a se sentir responsáveis e culpadas pelo aparecimento da dermatose. Fazer algo para controle dos sintomas requer que as circunstâncias, em especial as aversivas, responsáveis pelo que a criança está sentindo, sejam modificadas e que os comportamentos mais eficazes para lidar com elas sejam aprendidos. Contudo, essa aprendizagem será possível quando as mães forem capazes de identificar e descrever mais adequadamente tais situações, o que implica que deverão ter a oportunidade de aprender quais mudanças são necessárias, quando e como produzi-las (Gon et al., 2013).

Faz-se importante destacar que não se questiona aqui o sentir da criança enquanto um sofrimento genuíno que acontece mediante determinadas situações, como aquelas relatadas pelas mães, mas sim o papel causal desses sentimentos na manifestação e exacerbação dos sintomas. Embora a aparente proximidade da relação entre estresse emocional e inflamação cutânea na DA, por exemplo, tem levado à especulação de que o sistema nervoso simpático estaria envolvido na manifestação da doença, ainda não está cientificamente esclarecido se estados emocionais precedem a exacerbação dos sintomas e se a própria doença leva a um aumento da pressão psicológica para o indivíduo (Seiffert, Hilbert, Schaechinger, Zouboulis, \& Deter, 2005). Essa análise pode também ser considerada para o vitiligo e a psoríase.

\section{Considerações Finais}

Os resultados mostraram que as mães de crianças com doença crônica de pele avaliadas neste estudo identificaram os fatores ambientais e biológicos que desencadeiam cada uma das doenças. Independentemente do tipo de dermatose apresentado, os dados evidenciaram que situações de estresse agudo ou prolongado são apontados pelas mães como desencadeantes dos sintomas, sendo que a emoção também é destaca como diretamente relacionada à doença.
É provável que, mesmo em casos nos quais paciente e/ou seu cuidador identifiquem corretamente os fatores desencadeantes dos sintomas da doença de pele, como os descritos nas categorias 1 e 2, e apresentem alto nível de adesão, a melhora esperada não ocorra em decorrência de fatores situacionais e/ ou emocionais (Categorias 3, 4 e 5). Para essas mães que já avaliam sua criança apresentando problemas de comportamento correspondentes aos classificados pelo CBCL, a participação de outros profissionais da saúde é fundamental.

Foi possível, portanto, a partir deste estudo, avaliar não apenas se as mães tinham clareza quanto à etiologia, ao prognóstico e ao tratamento médico, como suas percepções sobre o aparecimento e a exacerbação dos sintomas. Contudo, existem limitações metodológicas nesta pesquisa que dificultam a identificação de outros fatores que podem contribuir para o resultado obtido e que deverão ser alvo de investigação em pesquisas posteriores. Outros instrumentos poderão ser incluídos para a avaliação comportamental dos cuidadores, como aqueles que avaliam estresse, ansiedade, depressão e práticas parentais. Isto seria importante, uma vez que a percepção dos pais a respeito do comportamento da criança como sendo problemático ou não, em alguns casos, pode estar sob o controle 1) de suas próprias práticas enquanto educador, 2) do seu próprio comportamento enquanto indivíduo e que foi aprendido ao longo de sua história, e/ou 3) do desconhecimento quanto ao que possa ser ou não um comportamento esperado para determinada faixa etária mediante situações de estresse e de ansiedade, por exemplo. Além disso, uma avaliação comportamental conduzida por meio do CBCL e de outros instrumentos direcionados a crianças e adolescentes poderá fornecer dados importantes sobre a interação destes com o cuidador e com a família. Mais especificamente, podem fornecer informações sobre se indivíduos com dermatoses crônicas percebem uma relação ou não entre a doença e o seu próprio comportamento, assim como as mães o fizeram no presente estudo. Poderia ser avaliado também o que as crianças e adolescentes conhecem sobre sua doença e tratamento e se identificam ou não fatores relacionados ao aparecimento e/ou exacerbação dos sintomas. Ainda, fatores que possam relacionarse aos resultados e que não foram analisados nesta pesquisa, como a idade e o sexo dos participantes e o tempo de diagnóstico médico dermatológico, podem ser investigados em estudos futuros e com uma amostra mais representativa desta população, além da inclusão de grupo controle com participantes que não tenham algum tipo de doença crônica de pele ou outra enfermidade. 


\section{Referências}

Achenbach, T. M. (1991). Manual for the Child Behavior Checklist/4-18 and 1991 Profile. Burlington: University of Vermont.

Alvarenga, T. M. M., \& Caldeira, A. P. (2009). Qualidade de vida em pacientes pediátricos com dermatite atópica. Jornal de Pediatria, 85(5), 415-420. http://dx.doi.org/10.1590/S0021-75572009000500008

Amaral, C. S. F. do, March, M. de F. B. P., \& Sant'Anna, C. C. (2012). Quality of life in children and teenagers with atopic dermatitis. Anais Brasileiros de Dermatologia, 87(5), 717-723. http://dx.doi.org/10.1590/S036505962012000500008

Barbarot, S., Gagnayre, R., Bernier, C., Chavigny, J. M., Chiaverini, C., Lacour, J. P., Dupre-Goetghebeur, D., Misery, L., Piram, M., Cuny, J. F., Dega, H., \& Stalder, J. F. (2007). Dermatite atopique: Un référentiel d'éducation du malade. Annals of Dermatology Venereology, 134(2), 121-127. http://dx.doi.org/10.1016/S0151-9638(07)91602-6

Barisić-Drusko, V., \& Rucević, I. (2004). Trigger factors in childhood psoriasis and vitiligo. Collegium Antropologicum, 28, 277-285. Recuperado de: http://hrcak.srce.hr/4906?lang=en

Ben-Gashir, M. A., Seed, P. T., \& Hay, R. J. (2002). Are quality of family life and disease severity related in childhood atopic dermatitis? Journal of the European Academy of Dermatology and Venereology, 16(5), 455-462. http://dx.doi. org/10.1046/j.1468-3083.2002.00495.X

Bordin, I. A. S., Mari, J. J., \& Caiero, M. F. (1995). Validação da versão brasileira do Child Behavior Checklist (CBCL) (Inventário de Comportamentos da Infância e Adolescência): Dados preliminares. Revista ABP-APAL, 17(2), 55-66.

Brufau, R. M., Berná, J. C., Redondo, C. B., Andreo, A. R., \& Gras, R. M. L. (2010). Estilos de personalidad en pacientes con psoriasis. Anales de Psicologia, 26(2), 335-340. Recuperado de: http://www.um.es/analesps/v26/ v26_2/18-26_2.pdf

Castro, E. K. de., \& Piccinini, C. A. (2002). Implicações da doença orgânica crônica na infância para as relações familiares: Algumas questões teóricas. Psicologia: Reflexão e Crítica, 15(3), 625-635. http://dx.doi.org/10.1590/ S0102-79722002000300016

Duarte, C. S., \& Bordin, I. A. S. (2000). Instrumentos de avaliação. Revista Brasileira de Psiquiatria, 22(2), 55-58. http:// dx.doi.org/10.1590/S1516-44462000000600015

Fagundes, A. J. F. M. (1983). Descrição, definição e registro de comportamento (3ำ ed.). São Paulo: Edicon.

Fontes, P. T. L., Neto, Weber, M. B., Fortes, S. D., Cestari, T. F., Escobar, G. F., Mazotti, N., Barzenski, B., Silva, T. L., Soirefmann, M., \& Pratti, C. (2005). Avaliação dos sintomas emocionais e comportamentais em crianças portadoras de dermatite atópica. Revista de Psiquiatria do Rio Grande do Sul, 27(3), 279-291. http://dx.doi.org/10.1590/S010181082005000300007

Gon, M. C. C., Gon, A. dos S., \& Zazula, R. (2013). Análise comportamental de relatos verbais de mães de crianças com dermatose crônica. Revista Brasileira de Terapia Comportamental e Cognitiva, 15(3), 57-71. Recuperado de: http:// pepsic.bvsalud.org/scielo.php?pid=S1517-55452013000300005\&script=sci_arttext

Gon, M. C. C., Rocha, M. M., \& Gon, A. dos S. (2005). Análise do conceito de estigma em crianças com dermatoses crônicas. Revista Brasileira de Terapia Comportamental e Cognitiva, 7(1), 15-20. Recuperado de: http://pepsic. bvsalud.org/scielo.php?pid=S1517-55452005000100004\&script=sci_arttext

Guibas, G. V., Gregoriou, S., Makris, M., Rigopoulos, D., \& Chliva, C. (2013). Atopic dermatitis, food allergy and dietary interventions: A tale of controversy. Anais Brasileiros de Dermatologia, 88(5), 839-841. http://dx.doi. org/10.1590/abd1806-4841.20132072

Kurizky, P. S., \& Mota, L. M. H. da. (2012). Disfunção sexual em pacientes com psoríase e artrite psoriásica: Uma revisão sistemática. Revista Brasileira de Reumatologia, 52(6), 938-948. http://dx.doi.org/10.1590/S048250042012000600011

Lipnharski, C., Bessa, G., Azevedo, P. A. d', Bonamigo, R. R., \& Quinto, V. P. (2013). Colonization by S. Aureus increases the EASI and the number of appointments by patients with atopic dermatitis: Cohort with 93 patients. Anais Brasileiros de Dermatologia, 88(4), 518-521. http://dx.doi.org/10.1590/abd1806-4841.20132046

Ludwig, M. W. B., Müller, M. C., Redivo, L. B., Calvetti, P. U., Silva, L. M. da., Hauber, L. S., \& Facchin, T. H. J. (2008). Psicodermatologia e as intervenções do psicólogo da saúde. Mudanças - Psicologia da Saúde, 16(1), 37-42. http:// dx.doi.org/10.15603/2176-1019/mud.v16n1p37-42

Manolache, L. (2011). The psychosocial aspects of vitiligo: A focus on stress involvement in children with vitiligo. In K. K. Park \& J. E. M. (Ed.). Vitiligo: Management and therapy (pp. 57-68). http://dx.doi.org/10.5772/25487

Manolache, L., Petrescu-Seceleanu, D., \& Benea, V. (2009). Correlation of stressful events with onset of vitiligo in children. Journal of the European Academy of Dermatology and Venereology, 23(2), 187-188. http://dx.doi. org/10.1111/j.1468-3083.2008.02764.x

Manzoni, A. P. D. da S., Nagatomi, A. R. da S., Townsend, R. Z., Pereira, R. L., Weber, M. B., \& Cestari, T. F. (2012). Assessment of the quality of life of pediatric patients with the major chronic childhood skin diseases. Anais Brasileiros de Dermatologia, 87(3), 361-368. http://dx.doi.org/10.1590/S0365-05962012000300002

Manzoni, A. P. D. da S., Nagatomi, A. R. da S., Townsend, R. Z., Weber, M. B., Pereira, R. L., \& Cestari, T. F. (2013). Assessing depression and anxiety in the caregivers of pediatric patients with chronic skin disorders. Anais Brasileiros de Dermatologia, 88(6), 894-899. http://dx.doi.org/10.1590/abd1806-4841.20131915 
Mesquita, K. de C., Costa, I. M. C., \& Igreja, A. C. de S. M. (2013). Atopic dermatitis and vitamin D: Facts and controversies. Anais Brasileiros de Dermatologia, 88(6), 945-953. http://dx.doi.org/10.1590/abd1806-4841.20132660

Nelms, B. C. (1989). Emotional behaviors in chronically ill children. Journal of Abnormal Child Psychology, 17(6), 657-668. http://dx.doi.org/10.1007/BF00917729

Palermo, E. C. (2010). Doenças de pele são agravadas durante o inverno. [On-line]. Recuperado de: $h t t p: / / w w w . s b c d$. org.br/pagina/1891

Pinquart, M., \& Shen, Y. (2011). Behavior problems in children and adolescents with chronic physical illness: A metaanalysis. Journal of Pediatric Psychology, 36(9), 1003-1016. http://dx.doi.org/10.1093/jpepsy/jsr042

Pires, M. C., \& Cestari, S. da C. P. (2005). Dermatite atópica. Rio de Janeiro: Diagraphic. http://dx.doi. org/10.1097/01206501-200506000-45

Rodrigues, R. N. dos S., Montealegre, F., Pires, M. C., Melo, J. F., \& Hahnstadt, R. L. (2011). Avaliação do teste de contato com aeroalérgenos em pacientes com dermatite atópica. Anais Brasileiros de Dermatologia, 86(1), $37-43$. http://dx.doi.org/10.1590/S0365-05962011000100004

Romiti, R., Maragno, L., Arnone, M., \& Takahashi, M. D. F. (2009). Psoríase na infância e na adolescência. Anais Brasileiros de Dermatologia, 84(1), 9-20. http://dx.doi.org/10.1590/S0365-05962009000100002

Rosa, E. C., \& Natali, M. R. M. (2009). Vitiligo: Um problema que não pode passar em branco. Revista Saúde e Pesquisa, 2(1), 119-126. Recuperado de: http://periodicos.unicesumar.edu.br/index.php/saudpesq/article/view/910/732

Sampaio, S. A. P., \& Rivitti, E. A. (1998). Dermatologia. São Paulo: Artes Médicas.

Sant'Anna, P. A., Giovanetti, R. M., Castanho, A., Bazhuni, N. F. N., \& La Selva, V. A. (2003). A expressão de conflitos psíquicos em afecções dermatológicas: Um estudo de caso de uma paciente com vitiligo atendida com o jogo de areia. Psicologia: Teoria e Prática, 5(1), 81-96. Recuperado de: http://www.mackenzie.br/fileadmin/Editora/Revista Psicologia/Teoria_e_Pratica_Volume_5_-_Numero_1/v5n1_art6.pdf

Schwartz, R., Sepúlveda, J. E., \& Quintana, T. (2009). Factores psicobiológicos en vitiligo infantil: Posible rol en su génesis e impacto en la calidad de vida. Revista Médica de Chile, 137(1), 53-62. http://dx.doi.org/10.4067/S003498872009000100008

Seiffert, K., Hilbert, E., Schaechinger, H., Zouboulis, C. C., \& Deter, H. C. (2005). Psychophysiological reactivity under mental stress in atopic dermatitis. Clinical and Laboratory Investigations, 210, 286-293. http://dx.doi. org $/ 10.1159 / 000084752$

Silva, C. M. de R., Gontijo, B., Pereira, L. B., \& Ribeiro, G. de B. (2007). Vitiligo na infância: Características clínicas e epidemiológicas. Anais Brasileiros de Dermatologia, 82(1), 47-51. http://dx.doi.org/10.1590/S036505962007000100006

Silva, J. D. T. da., \& Müller, M. C. (2007). Uma integração teórica entre psicossomática, stress e doenças crônicas de pele. Estudos de Psicologia, 24(2), 247-256. http://dx.doi.org/10.1590/S0103-166X2007000200011

Silva, M. F. P. da, Fortes, M. R. P., Miot, L. D. B., \& Marques, S. A. (2013). Psoriasis: Correlation between severity index (PASI) and quality of life index (DLQI) in patients assessed before and after systemic treatment. Anais Brasileiros de Dermatologia, 88(5), 760-763. http://dx.doi.org/10.1590/abd1806-4841.20132052

Souza, A. P. F. dos S., Carvalho, F. T., Rocha, K. B., Lages, M. N., Calvetti, P. U., \& Castoldi, L. (2005). Associação de eventos estressores ao surgimento ou agravamento de vitiligo e psoríase. Psico, 36(2), 167-174.

Tates, K., Elbers, E., Meeuwesen, L., \& Bensing, J. (2002). Doctor-parent-child relationships: A 'pas de trois'. Patient Education and Counseling, 48(1), 5-14. http://dx.doi.org/10.1016/S0738-3991(02)00093-9

Tejada, C. dos S., Mendoza-Sassi, R. A., Almeida, H. L. de., Jr., Figueiredo, P. N., \& Tejada, V. F. dos S. (2011). Impact on the quality of life of dermatological patients in southern Brazil. Anais Brasileiros de Dermatologia, 86(6), 1113-1121. http://dx.doi.org/10.1590/S0365-05962011000600008

Torres, R. A. T., Silva, S. A. da, Magalhães, R. F., Morcillo, A. M., \& Velho, P. E. N. F. (2011). Comparação entre questionários de qualidade de vida e sua correlação com a evolução clínica de pacientes com psoríase. Anais Brasileiros de Dermatologia, 86(1), 45-49. http://dx.doi.org/10.1590/S0365-05962011000100005

Weber, M. B., Lorenzini, D., Reinehr, C. P. H., \& Lovato, B. (2012). Assessment of the quality of life of pediatric patients at a center of excellence in dermatology in southern Brazil. Anais Brasileiros de Dermatologia, 87(5), 697-702. http:// dx.doi.org/10.1590/S0365-05962012000500004

\section{Autores:}

Natália Guimarães Dias - Mestre, Faculdade de Filosofia, Ciências e Letras de Penápolis.

Márcia Cristina Caserta Gon - Doutora, Universidade Estadual de Londrina.

Caroline Audibert Henrique - Mestranda, Universidade Estadual de Londrina.

Lorrana Muriéli Araújo Barros - Mestranda, Universidade Estadual de Londrina.

Taís da Costa Calheiros - Mestranda, Universidade Estadual de Londrina.

\section{Endereço para correspondência:}

Rodovia Celso Garcia Cid (PR 445), km 380

Caixa Postal 6001

86051-990 Londrina, PR, Brasil

Recebido em: 08.12.2014

Aceito em: 21.01.2016 